Cet article touche à un sujet qui est important pour une grande partie du corps médical. Les avis qui y sont exprimés reflètent les opinions de son auteur, qui ne recouvrent pas obligatoirement celles de la rédaction.

La rédaction

\title{
Effets pervers de la statistique mise au point par santésuisse pour le contrôle de l'économicité des traitements
}

\author{
3,5 milliards de francs jetés par la fenêtre
}

\section{H. Siegenthaler}

\section{Introduction}

Le contrôle du travail des médecins institué par santésuisse pèse, depuis 15 ans, très lourdement sur le corps médical. Il nous parait nécessaire de faire le point.

Avant tout, force est de constater que ce contrôle n'est parvenu ni à réduire, ni même à stabiliser les coûts sanitaires, au contraire: bien que d'autres facteurs contribuent à leur augmentation, il est indéniable qu'il y concourt massivement.

Il n'est pas pratiqué dans tous les cantons avec la même intransigeance brutale et il semble que l'agressivité des exécutants soit inversement proportionnelle à la valeur du concept sur lequel il se fonde. Dans le canton de Berne, par exemple, ou il ne repose sur aucun concept précis, il est exercé avec une balourdise hors du commun.

Plusieurs études portant sur le contrôle exercé par santésuisse sont parvenues à la conclusion qu'il pouvait avoir des effets pervers tels le cloisonnement de la médecine, les hospitalisations inutiles [1, 4], l'augmentation des fautes d'art, le fait que les cas lourds sont négligés [2], ainsi qu'une charge administrative excessive.

Compte tenu de tous les inconvénients qu'elle comporte, la statistique de santésuisse coûterait, par année, quelques 3,5 milliards de francs aux assurés en dépenses inutiles.

\section{Le contrôle d'économicité: un leurre}

Correspondance:

Dr Henri Siegenthaler Chemin de la Passerelle 24 CH-2503 Bienne
Un des principaux défauts du contrôle de l'économicité appliqué au travail des médecins est de n'avoir qu'une logique comptable [3].
Les autorités fédérales devraient se rendre compte que c'est la une approche insuffisante: la qualité d'une prestation ou d'un produit doit forcement être prise en considération dans tout contrôle sérieux. Les coûts en soi ne sauraient constituer un critère d'évaluation s'ils ne sont pas mis en rapport avec la qualité.

Un contrôle qui tiendrait compte de la qualité serait évidemment à l'avantage des assurés, et surtout des patients.

Or, pour des raisons «de simplification», le chef du Département fédéral de l'intérieur (DFI) et l'Office fédéral de la santé publique (OFSP), font abstraction du rapport qualité - prix, suivis en cela par santésuisse et par l'Office fédéral des assurances sociales (OFAS). Prétexte invoqué: les intéressés ne parviendraient pas à trouver un critère d'évaluation de la qualité!

\section{Aberrations de la méthode statistique de santésuisse}

La méthode statistique élaborée par santésuisse compare notamment les coûts annuels occasionnés par les médecins spécialistes d'une même discipline pour les patients traités dans les divers cabinets médicaux mais ne prend pas en considération, ou dans une très faible mesure seulement, les coûts générés à l'extérieur. Le but est de dépister les médecins qui dépassent la limite annuelle de coûts au cabinet admise, fixée selon une indexation arbitraire. Les praticiens qui dépassent cette limite sont remis à l'ordre et incités à dispatcher leurs patients ou à les hospitaliser. 


\section{Une différence entre médecins n'existerait pas}

Cette méthode se fonde donc sur un principe impliquant que tous les praticiens d'une même discipline font une même médecine et ont une clientèle comparable: on est bien loin de la réalité. Pourtant, cette pratique erronée est entérinée par les tribunaux, en particulier par le Tribunal fédéral des assurances (TFA), aussi surprenant que cela puisse paraître.

L'importance du groupe de référence, dans toute étude comparative, n'échappe à personne. Les membres de toute discipline médicale, en effet, ont chacun leur manière de travailler et évoluent différemment. Par conséquent, ils auront tous une clientèle différente. Or, les médecins sélectionnés pour servir de référence l'ont été sans que les critères appliqués dans ce choix aient pu être déterminés.

En outre, santésuisse prétend tenir compte, pour parfaire la comparabilité, du genre de lieu où sont situés les cabinets médicaux, puisque les habitudes peuvent changer, de l'un à l'autre, suivant certaines caractéristiques sociologiques.

Or, sa méthode simpliste, usant d'un principe fédéraliste qui date d'un autre temps, suit les frontières cantonales, car selon santésuisse, les variations observées en ce qui concerne les besoins médicaux des populations sont influencées par l'appartenance de celles-ci aux divers cantons. Ce qui n'empêche pas les assureurs du canton de Berne, par exemple, de fixer les montants des primes suivant une répartition en trois zones, en fonction des comportements différents existant dans chacune d'elles (villes, faubourgs, campagne). En revanche et contre toute logique, les médecins, où qu'ils pratiquent, sont mis dans le même panier, ce qui est d'ailleurs contraire aux méthodes mêmes de la statistique de santésuisse.

\section{Prestations contingentées}

La statistique en soi peut être exempte d'erreurs, les chiffres exacts, la saisie des données sans faille: ce n'est pas suffisant toutefois pour répondre aux exigences de la loi.

Du reste, les résultats de santésuisse sont mis à mal par une statistique identique qu'a effectuée un groupe de médecins. L'erreur imputable à santésuisse serait très importante.

L'article 56 LAMal demande au prestataire de soins de limiter sa prestation à ce qu'exigent l'intérêt de l'assuré et le but du traitement. Le but du traitement est déterminé par le diagnostic et l'intérêt de l'assuré, par la guérison de celui-ci ou par l'obtention, par lui, d'une qualité de vie acceptable.

L'esprit de la loi n'est respecté ni par le DFI, ni par santésuisse, qui réduisent les problèmes sanitaires à un problème pécuniaire [3], en contingentant les soins que les médecins sont autorisés à accorder à chacun de leurs patients. Ces pratiques, qui rappellent celles qui ont cours dans l'agriculture, excluent tout esprit d'émulation et de concurrence, ce qui est en soi une conséquence désastreuse.

\section{Analyse sectorielle, refus d'une analyse globale}

Mais le principal défaut de la méthode statistique de santésuisse est certes de ne pas indiquer ce que coûte globalement un assuré par année, puisque seuls les coûts générés dans les cabinets médicaux sont pris en considération à l'exception de quelques postes mineurs. Ne sont donc pas pris en compte ceux qui sont induits à l'extérieur et qui représentent une partie très importante de dépenses comme l'imagerie médicale, les traitements dans les centres spécialisés, les hospitalisations et les prestations de spécialistes. L'image sectorielle ainsi donnée ne correspond donc en rien à la réalité.

Santésuisse et l'OFAS ne se soucient pas de savoir si le travail du médecin est adéquat et réellement économique: leur seul objectif est d'attribuer à chaque cabinet médical un contingent de prestations par patient et par année.

Cette manière de favoriser le cloisonnement de la médecine et de rendre impossible la prise en charge globale d'un patient est inhumaine.

Les effets pervers de la méthode utilisée par santésuisse ont été démontrés à la faveur de plusieurs études effectuées par des experts chevronnés.

\section{Effets pervers de la méthode statistique de santésuisse}

Le contingentement des soins oblige les médecins, d'une part, à éviter de soigner les cas lourds, les malades chroniques, afin d'éviter les sanctions sévères qu'ils risquent, et d'autre part, à multiplier les hospitalisations. 6 à 7\% des hospitalisations, bien que toutes ordonnées par des médecins, seraient inutiles. Il s'agirait de quelque 500 millions de francs dépenses en pure perte $[1,4]$. 
En 2000 déjà, les professeurs André Dubey et François Dufresne [5], dans leur étude effectuée à l'université de Lausanne, attiraient l'attention sur les effets négatifs que pourraient avoir la méthode statistique de santésuisse.

Il ressort de diverses études que les médecins traitent en moyenne, par patient, 1,5 atteintes diagnostiquées. Dans le concept de la prise en charge globale, les mêmes statistiques montrent que ce sont, en moyenne, plus de trois problèmes différents auxquels il est remédié en même temps. Les pratiques de santésuisse poussent les médecins à confier à des collègues les traitements supplémentaires ou à hospitaliser les patients. Sachant que les dépenses médicales totales se montent à 6,5 milliards de francs par année, on peut estimer à 2 milliards de francs au moins les dépenses inutiles occasionnées par la méthode de santésuisse laquelle oblige les médecins à une prise en charge sectorielle et à dispatcher.

La minutie apportée aux contrôles par santésuisse et la nécessité de vérifier des millions de factures dont l'ampleur et la teneur sont des plus diverses, l'obligation de tout mettre sur ordinateur afin de connaître le nombre exact des injections faites, des pansements utilisés, des moindres gestes médicaux, conduit à une bureaucratie hors de toute proportion. Le professeur Sheldon s'est attaché à analyser l'efficience de l'administration des diverses caissesmaladie [6]. Il est arrive à la conclusion qu'actuellement, la mauvaise administration des caisses-maladie coûtait chaque année environ 1 milliard de francs aux assurés en dépenses inutiles.

Ce qui est particulièrement grave, c'est que suite au contingentement des soins, les praticiens qui prennent en charge les cas lourds, générant des coûts importants, tels les cas de sida, d'hépatite C, la sclérose en plaque, les maladies auto-immunitaires, etc., sont dénoncés comme favorisant la surconsommation médicale et assignés devant les tribunaux, tous partisans du système en place, ainsi que le démontre l'article de madame Edith Lier [7].

Même les sociétés médicales s'inquiètent de ce dérapage de la méthode de contrôle de santésuisse (cf. l'article paru dans le Bulletin des médecins suisses en 2003: Ärzte werden für die umsichtige Betreuung von Langzeitkranken finanziell bestraft [«Des sanctions financières punissent les médecins qui prennent en charge des patients atteints de maladies de longue durée»]) [2].

\section{Euthanasie passive et augmentation des erreurs médicales}

Sous la pression des caisses-maladie, certains médecins renonceront forcément à prendre en charge des patients nécessitant des traitements lourds ou, ce qui est pire, leur offriront en connaissance de cause une médecine de qualité inférieure, se rendant ainsi coupables d'euthanasie passive.

Enfin, l'article paru le 27 mai 2005 dans la SonntagsZeitung doit être pris très au sérieux: Eine längst fällige Operation [8]. Il fait état de manière dramatique d'une augmentation inquiétante des cas d'erreurs médicales et du nombre des procès intentés à des médecins pour négligence ou traitement erroné. L'OFAS avait déjà, il y a quelques mois, souligné l'augmentation des cas de pratiques erronées dans les hôpitaux et les cabinets médicaux.

Que les médecins ne travaillent plus selon l'art, mais dans le seul souci d'éviter les sanctions promises au moindre dépassement de l'index est donc le résultat de la terreur qu'exerce santésuisse sur le corps médical au moyen de sa méthode statistique malvenue.

\section{Une médecine dépourvue d'humanité?}

Selon une logique purement comptable [3] le système de contrôle de la statistique de santésuisse sert à établir et à attribuer un contingent de prestations que le médecin est en droit de fournir par année à chacun de ses patients. Elle est aussi justement utilisée pour dépister les médecins qui coûtent chers. Or, ce sont eux qui traitent les patients souffrant de pathologies lourdes et qui nécessitent évidemment des traitements plus chers.

Ceci est clair pour tout le monde, et donc pour la classe politique également, que les cas lourds nécessitent les traitements les plus chers.

Quant aux raisons de cette campagne contre les médecins qui traitent les pathologies lourdes, elles peuvent être d'ordre divers. S'agit-il simplement d'un dérapage d'une bureaucratie incontrôlée et de l'application, sans distinction aucune, de certaines normes par un personnel obtus et mal formé? Ou d'une décision politique délibérée visant, dans un premier temps, à débarrasser les assureurs des «mauvais risques» et, dans un second temps, à décharger les assurances sociales du poids que représente, pour elles, cette catégorie de malades (ce qui équivaudrait à l'instauration d'une euthanasie indirecte)? 
En fixant une norme à ne pas dépasser, en matière de soins, les responsables n'ont pas tenu compte du progrès constant de la médecine laquelle se développe pratiquement de jour en jour. Toute nouveauté qui pourrait être utilisée par un médecin avant les autres, moyennant évidemment, un coût forcement plus élevé, est d'emblée écartée; le médecin est prié de ne pas prétendre se placer à la pointe du progrès.

Curieuse contradiction: d'une part, on encourage et l'on favorise la formation continue, qui va sans doute au delà de ce qui serait nécessaire, et d'autre part, on sanctionne très gravement les médecins qui appliquent les nouvelles méthodes de traitement, n'hésitant pas à les ruiner ou à les exclure de la pratique médicale.

Il n'est pas étonnant que les praticiens, soumis à une telle terreur, pourraient sous la pression en arriver à négliger leur travail, ce qui pourrait conduire à des erreurs professionnelles.

\section{L'avenir}

Il semble bien qu'il n'y ait aucune amélioration à attendre du quarteron de fanatiques qui veillent à l'application de la méthode statistique instituée par santésuisse. Néanmoins, un article paru dans Infosantésuisse permet de supposer que ceux qui détiennent le pouvoir de décision se rendant compte des effets pervers du système imposé; ils insistent sur trois points:

1. aucun médecin ne devrait plus être contraint au remboursement des coûts de traitement lorsqu'il a accepté de soigner des cas lourds;

2. les groupes de référence doivent être constitués correctement, en fonction des particularités des médecins et de leur clientèle, ainsi que de la situation géographique des cabinets médicaux;

3. le travail de chaque prestataire de soins doit être soumis à une analyse approfondie.
Pour l'instant, ces idées nouvelles n'ont pas été reçues chez ceux qui devraient les appliquer.

Si cette méthode statistique devait être maintenue, il conviendrait pour protéger les patients d'inviter les décideurs de santésuisse, de l'OFAS et du DFI à obliger les médecins à livrer les diagnostics précis établis dans chaque cas et, sur la base de ces indications, à établir une statistique des coûts totaux par cas ou par diagnostic. Ce serait la seule manière d'obtenir que les contrôles soient effectués en fonction du rapport qualité/ coût, comme il est d'usage de le faire dans tous les domaines, celui de la médecine constituant la seule exception à cette règle.

\section{Références}

1 Revue des Hospitalisations: Département de la santé, des affaires sociales et de l'énergie du canton du Valais, 5 avril 2000.

2 Gesellschaft der Ärztinnen und Ärzte des Kantons Solothurn. Ärzte werden für die umsichtige Betreuung von Langzeitkranken finanziell bestraft. Schweiz Ärztezeitung 2003;84(50):2664-5.

3 Hurst Samia A, Mauren A. Selectiva contacting of Swiss physicians: ethical issues and open questions. Swiss Med Wkly 2004;134:632-9.

4 Müller R. Unnötige Einweisungen kosten schweizweit mindestens eine halbe Milliarde Franken pro Jahr. SonntagsZeitung, 11 octobre 2004.

5 Dubey A, Dufresne F. Etude du contrôle du caractère économique des traitements basé sur les statistiques du Concordat des assureurs maladie suisses. HEC-Universite de Lausanne, février 2000.

6 Sheldon G. Die Kosteneffizienz der Schweizer Krankenversicherungen 1994/2001. Schlussbericht zu einem Forschungsprojekt im Rahmen des Schweizerischen Nationalen Forschungsprogramms 45. FAI Universität Basel. 9 janvier 2004.

7 Lier E. Kassen treiben Ärzte in den Ruin, Beobachter 2004;16:26-7.

8 Nientit C. Eine längst fällige Operation. SonntagsZeitung, 29 mai 2005. 